\title{
An Evaluation of Accessibility of Hierarchical Data Structures in Data Broadcasting -Using Tactile Interface for Visually-Impaired People-
}

\author{
Takuya Handa, Tadahiro Sakai, Kinji Matsumura, \\ Yasuaki Kanatsugu, Nobuyuki Hiruma, and Takayuki Ito \\ NHK Schience \& Technical Research Laboratories, \\ 1-10-11, Kinuta, Setagaya-ku, Tokyo, Japan \\ \{handa.t-es, sakai.t-jk, matsumura.k-ke, kanatsugu.y-ki, \\ hiruma.n-dy, itou.t-gq\}@nhk.or.jp
}

\begin{abstract}
We have been developing a barrier-free information receiving system for the purpose of communicating information in digital broadcasting to visually-impaired people. In the service of data broadcasting in digital broadcasting, many items constitute the menu screen. In this report, presentation methods and access methods using touch in combination with audio for the purpose of effective communication of menu screen structure are briefly explained. Secondly, results of evaluation experiments conducted to obtain a guideline to design hierarchical presentation structures easily accessible by visually impaired people, using the tactile interface in combination with audio presentation were discussed by focusing on the hierarchical structure of menu screens in data broadcasting.
\end{abstract}

Keywords: accessibility, hierarchical structure, data broadcasting, tactile interface, visually-impaired.

\section{Introduction}

TV is one of the most basic methods to provide information in our society; therefore it is an important task to develop an information presentation method as well as an access method easily understood by everyone. The authors of this paper have been developing a barrier-free information receiving system for the purpose of communicating information in digital broadcasting to visually-impaired people including those totally blind, with weak vision and blind-deaf. We are also researching optimum information presentation methods as well as access methods based on information reception and recognition [1]. In the service of data broadcasting in digital broadcasting, many items including news and weather reports constitute the menu screen. In addition, since the menu screen has a hierarchical display in many cases, it is not easy for visually impaired people to promptly understand the screen composition and hierarchical structure by audio guidance only to access desired information [2][3]. It is therefore implied that parallel use of the 
audio presentation and tactile presentation is effective for understanding the spatial structure of the screen. [4][5]. In this report, presentation methods and access methods utilizing touch for the purpose of effective communication of menu screen structure and electronic program guide (EPG) in data broadcasting are briefly explained. Secondly, results of evaluation experiments conducted to obtain a guideline to design easily accessible contents by visually impaired people, using tactile interface in combination with audio presentation were discussed by focusing on the hierarchical structure of menu screen in data broadcasting.

\section{Hierarchical Structures and Presentation Methods in Data Broadcasting and the Web}

Data broadcasting and EPG are operated with GUI that accesses information by selecting a desired item on the screen. In many cases, each item in the menu screen in data broadcasting is indicated hierarchically by category. In the case of the PC and the Web are composed of complicated hierarchy or nesting. For access methods of PCs for visually impaired people, the interface with sequential information using arrow key operation and audio (audio navigation) is the mainstream. However, it is not easy for visually impaired people to understand the screen composition and its hierarchical structure by audio guidance only. Especially for the TVs, it is an important task to develop information presentation and access methods easily understood by everyone. We therefore have been developing a barrier-free information receiving system. In the previous studies on the accessibility of hierarchical structures, it is pointed out that the factors such as the overall site structure and understandability of labels would influence the accessibility [6]. On the other hand, it is indicated that search methods with tactile display are effective because they make search paths fewer and estimating approximate position of target objects easier [4]. As an example of tactile navigation method that use touch in combination with audio presentation, we have developed an array-type tactile interface that incorporates tactile keys having an projectile/retractile mechanism. We call the proposed system Tactile Navigator [1].

\section{Communication of Menu Screen Structure in Data Broadcasting by the Tactile Navigation}

\subsection{Composition and Moving Function of Tactile Navigator}

In Tactile Navigator (Figure 1), vertically moving switches (tactile keys) are installed in the arrangement of telephone buttons because it is known that the arrangement is suitable for daily use, reflecting the results of the preliminary experiments. In the preliminary experiments, time required for key search was obtained depending on the arrangement of tactile keys as well as the height of keys. As a result, the search time was significantly shorter in the case of the arrangement of telephone buttons than in the case of one horizontal column such as numerical keys in a full keyboard, 


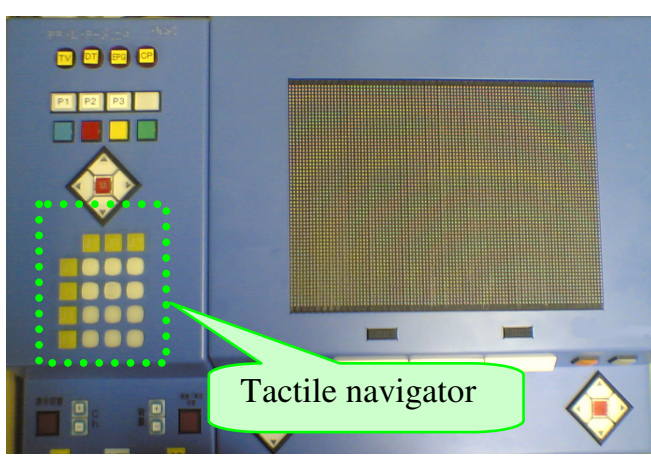

Fig. 1. Tactile Presentation Terminal and the best height of keys was approximately $3 \mathrm{~mm}$.

In this developed system, users are able to know the selectable items in the GUI screen as well as composition of cells in a table such as EPG, by touching the presentation surface of Tactile Navigator and understanding the arrangement, shape and the number of tactile keys. By pressing down a tactile key, the content can be confirmed by audio or Braille, to access information [1].

\subsection{Tactile Presentation of Menu Screen Composition in Data Broadcasting}

Methods to present items originally in a GUI screen onto a tactile display include a screen presentation method which faithfully presents the shape of screen items as well as a logical presentation method which presents the items by corresponding them to other objects with different abstract shapes and sizes for easy tactile recognition [1]. As shown in Figure 2, Tactile Navigator is a logical presentation method. The figure shows an example of the operation of Tactile Navigator. The raised circular tactile keys S1-S7 represent the number of selectable items, and the raised square tactile keys N1-N3 represent levels in the menu hierarchy. The remaining tactile keys (shown as dotted lines) are at the same height as the background surface. By using differently shaped key for the selectable items and for the hierarchical levels, these different capabilities can be easily differentiated by touch. For example in Figure 2, the current location is in the third depth of the hierarchy, indicating that there are seven selectable items. It is also possible to directly transit among layers with the hierarchical level keys [1].

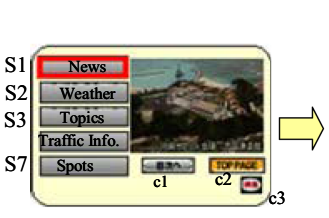

An example of data -broadcast menu screen

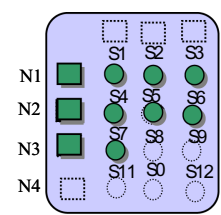

(a) Presentation method by array type tactile interface

Nn :Layer presentation Sn :selectable items

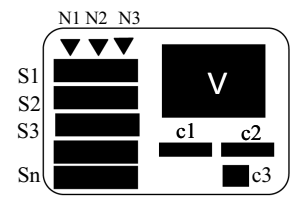

(b) Screen presentation method by tactile display

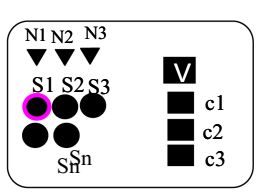

(c)Logical presentation method by tactile display

Fig. 2. Presentation method for selectable items and layer structure using tactile unit

\section{Evaluation of Accessibility of Hierarchical Structures}

\subsection{Purpose of Experiments}

According to the previous experiments, the tactile navigation proposed by the authors was found to be able to considerably decrease the time to search desired items and to 
be effective for accessibility improvement compared to the audio navigation. Furthermore, based on introspective reporting by the subjects, it has been indicated that the tactile navigation is effective not only in terms of operability and accessibility but also for understanding the composition of menu screen or tables in data broadcasting [1]. However, the previous study was conducted on one specific hierarchical structure. Therefore it is necessary to examine more general cases. The purpose of this experiment is to clarify the hierarchical structure easily accessible to menus in data broadcasting. Thus, by focusing on the hierarchical structure of menus in data broadcasting, we conducted evaluation experiments to obtain a guideline for hierarchical presentation easily understood and accessible by visually impaired people.

\subsection{Evaluation Experiments in Regard to Presentation of Hierarchical Structures}

\section{Experimental Method and Conditions}

In the experiments, we evaluated differences in the search time and the number of operations for items given randomly by the audio navigation and the tactile navigation to hierarchical structures with different numbers of layers and branches. The experimental conditions are described in Table1.

Table 1. Experimental conditions

\begin{tabular}{llll}
\hline \multicolumn{1}{c}{ Factors } & \multicolumn{2}{c}{ Levels } \\
\hline Presentations method & \multicolumn{2}{c}{ Audio navigation } & Tactile navigation \\
\hline \multirow{2}{*}{ Hierarchical structure } & 4- branches & 8-branches & 16-branches \\
& 4-layers & 3-layers & 2-layers \\
\hline
\end{tabular}

With hierarchical structures and presentation methods as evaluation factors, conditions included three patterns of hierarchical structures and two presentations methods: the audio navigation and the tactile navigation. The simulated structure of data broadcasting menu screens with 4- branches/4-layers used for the experiment is shown in Figure 3. The hierarchical structure was given four layers at maximum, considering the number of layers presented in typical data broadcasting. With the total number of items in the deepest layer (the number of terminal nodes) as 256, three patterns were prepared so that the number of branches equaled $2^{\mathrm{n}}$ (Table 2). In regard to the 8-branches/3-layers, there are four items only for the third layer, in order to make the number of terminal nodes 256 . The variety in the total number of nodes was not adjusted in this experiment.

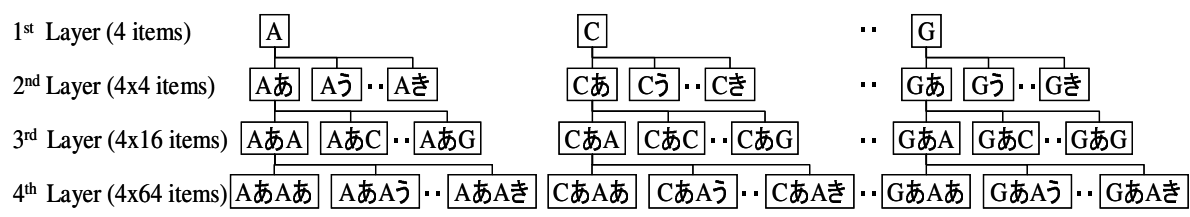

Fig. 3. Example of the hierarchical structure for evaluation experiment 
Table 2. Hierarchical structures for experiment

\begin{tabular}{clc}
\hline & Number of terminal nodes & Total number of nodes \\
\hline 4- branches/4-layers & $256(4 \times 4 \times 4 \times 4)$ & 340 \\
8- branches/3-layers & $256(8 \times 8 \times 4)$ & 328 \\
16- branches/2-layers & $256(16 \times 16)$ & 272 \\
\hline
\end{tabular}

Items in each hierarchy were composed by the combination of letters of "alphabet" and "hiragana," which were lined up alternatively one letter after another starting from the first letter. As the layer goes deeper, alphabet and hiragana were connected alternatively so that the layer and item can be uniquely identified with a given task. We asked to search an item given randomly without limiting to terminal nodes, and evaluated the understandability and accessibility of hierarchical structures according to the search time and the number of operations. The starting point was the first item in the first layer for the first task of each experimental condition, or the item at the time of completion of a previous task for other cases. The interval between the point when a task was given and the point of task completion was measured as the search time. We did not give subjects instruction such as "as quickly as possible" or "fewest operations as possible" but asked to search freely.

In the experiments, search tasks with 36 items were conducted for all six combinations of factors and levels indicated in Table 1 . With this 216 (36 x 6) trials as one cycle, experiments were repeatedly conducted five times, and learning effects to the hierarchical structure were also evaluated. The subjects included two visually impaired people and two non-visually-impaired people (Table 3). The non-visuallyimpaired people were blindfolded upon experiments.

Table 3. Subject profiles

\begin{tabular}{cccc}
\hline Subject & Sex & Age & Lost sight era \\
\hline A & F & 20 & Sighted \\
B & F & 20 & Sighted \\
C & F & 20 & 10 \\
D & F & 50 & 10 \\
\hline
\end{tabular}

\section{Experimental Results}

Regarding the search time and the number of operations, we conducted an analysis of variance (ANOVA) based on a two-way layout using "presentation method" and "hierarchical structure" as the factors. The main effect of each were significant at a $1 \%$ level. In Figure 4, 95\% confident interval of the sample mean and population mean of the number of operations are shown in terms of hierarchical structures. According to this result, there was a tendency mostly consistent with the theoretical minimum numbers of operations in the tasks. The difference in the number of operations between the audio navigation and the tactile navigation was significant for more branches and fewer layers. 


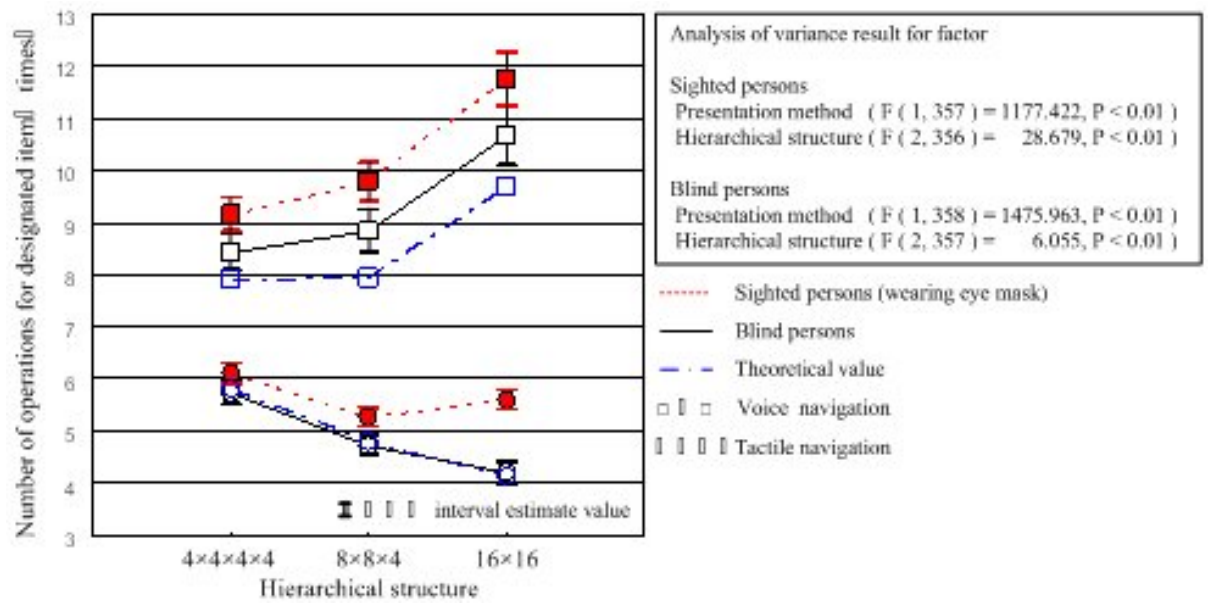

Fig. 4. Number of operations depend on presentation methods and hierarchical structures

Figure 5 shows the results on the search time. In regard to the search time, very similar tendency was observed for both non-visually-impaired people and blind people although the absolute values were different, and tactile navigation with 8branches/3-layers had the shortest time. The difference in the search time between the audio navigation and the tactile navigation was smaller for more branches and fewer layers. The theoretical value of the shortest operation and search time indicated in the figure assumes that one second is the minimum time necessary for one mechanical operation, representing the product of the minimum time and the number of
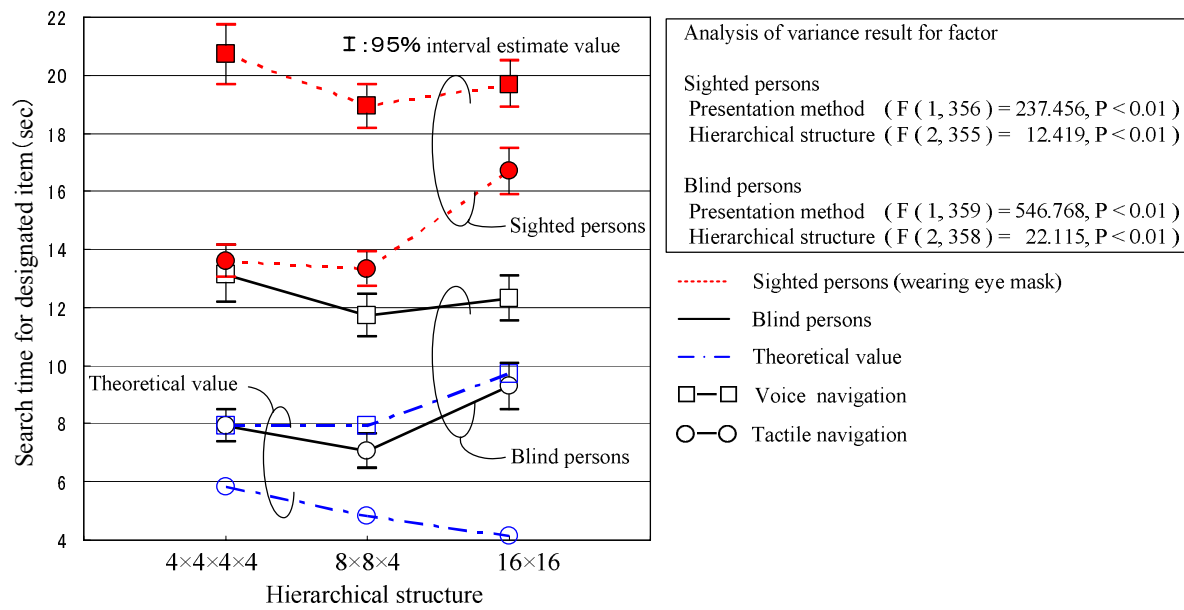

Fig. 5. Search time depend on presentation methods and hierarchical structures 


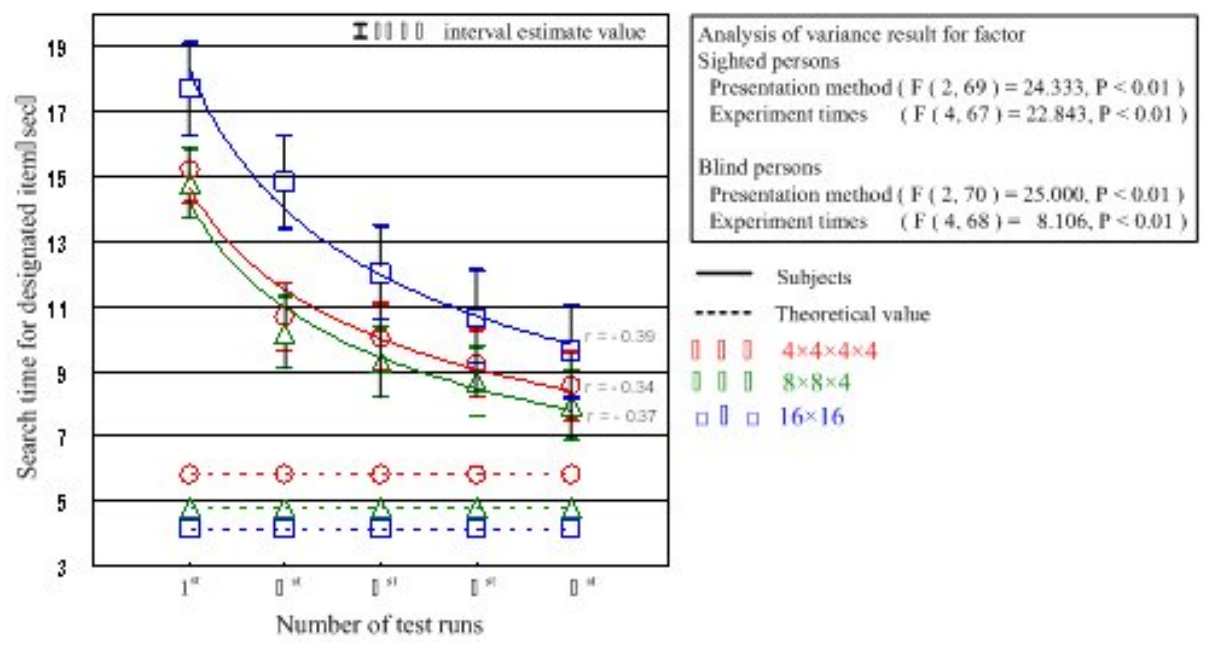

Fig. 6. Search time and learning effect depend on hierarchical structures by using tactile navigation

operations for the shortest route in the given task. Thus, it means the theoretically shortest time in the case that the task is performed with the shortest route without any thinking intervals.

We conducted an ANOVA of the search time treating the hierarchical structure and number of test runs as a two-way layout. The main effects of each were significant at a $1 \%$ level. In Figures 6, the horizontal axis shows the number of test runs, and the search time is expressed for each hierarchical structure.

\section{Discussion}

As a result of evaluation of the influence to the search time and the number of operations by factors of presentation methods and hierarchical structures, both the number of search operations and the length of search time were significantly smaller in the case of the tactile navigation than the audio navigation. This is consistent with the results of evaluation experiments conducted previously [1]. We now discuss understandability and accessibility of the hierarchical structures.

\section{Accessibility of hierarchical structures}

According to the results in Figure 5, the search time for 8-branches/3-layers was the shortest for both presentation methods. The 8-branches/3-layers condition is considered as the most accessible structure handled in this experiment. However, according to the prediction from theoretical values in this task, it was expected that 4branches/4-layers and 16-branches/2-layers would have the shortest search time for the audio navigation and the tactile navigation respectively. Contrary to the prediction, however, 4-brances/4-layers and 16-branches/2-layers had the longest search time for the audio navigation and the tactile navigation, respectively. From 
Figure 3, results on the number of operations was consistent with theoretical values in both cases, and it should be noted that increase of the number of operations does not simply cause the lengthening of the search time.

As a factor that caused the longest search time for 4-branches/4-layers in the audio navigation, it is considered that an increase of the number of layers made it difficult to understand the current location and the overall hierarchical structure just by audio presentation. Based on this, it seems that a transition among layers required more time for a decision than a transition among items, which is conducted with the one operation. On the other hand, as a factor that caused the longest search time for 16branches/2-layers in the tactile navigation, it is considered that the arrangement of items could not be understood and the advantage of direct switching could not be utilized. This seems to be related to the fact that the number of items far exceeds the magic number $(7 \pm 2)$ for short-term memory.

As described earlier, despite the fact that the number of operations did not increase even if the number of hierarchies or branches was large (selection was hardly mistaken), the search time for the above two cases was extremely long, which can be considered as the results of longer thinking time. This can be considered as the psychological influence of attempting to memorize the arrangement of items or to avoid unnecessary operations, as well as the influence of the fact that the order of items could be deduced in this task due to its regularity. Further study will be necessary concerning factors depending on users' preferences.

\section{Effects of tactile navigation}

In Figure 7, the difference in the search time between the audio navigation and the tactile navigation was described according to the results of Figure 5. This implies that the more different the search time is between the audio navigation and the tactile navigation, the higher the effect of the tactile navigation will be in the hierarchical

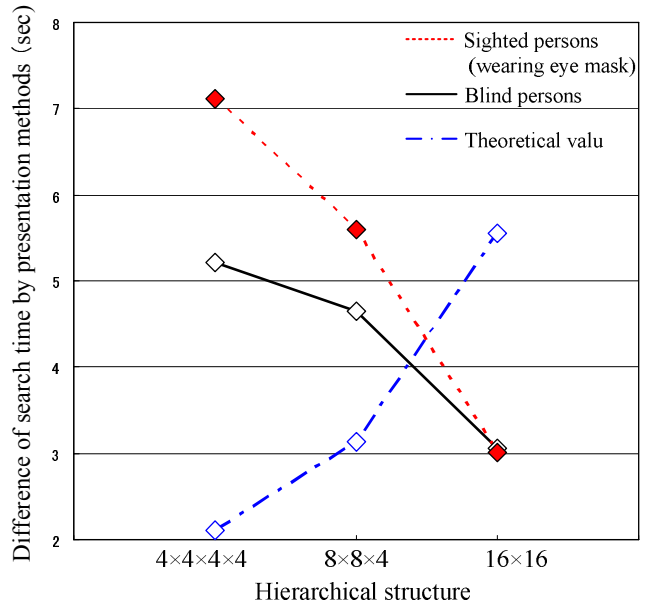

Fig. 7. Difference of search time by presentation methods depend on hierarchical structures structure. Based on the results, 4branches/4-layers was the most effective for the tactile navigation and 16-branches/2-layers was the least. This is considered to imply that the tactile navigation is effective to understand current locations as well as hierarchical structures. Furthermore, in the experiment, cases such that a current location was confirmed several times or the search was continued after returning to the top layer were observed for the audio navigation. On the contrary, according to the introspective reporting by the subjects, the hierarchical structure was easy to understand by utilizing touch. However, it is hard to judge from this experiment whether this effect of the tactile navigation 
depends on the advantage of direct switching between items/layers or on the tactile presentation itself clarifying the number of items or the current layer position.

\section{Learning effects}

The learning curve expressing learning effects can be expressed as the following equation by assuming a negative acceleration type [7].

$$
\mathrm{y}=\mathrm{A}-\left(\mathrm{A}-\mathrm{y}_{0}\right) \exp (-\mathrm{rx})
$$

A: Asymptotic value (limitation of learning)

$\mathrm{y}_{0}$ : Initial value of learning

r: Learning rate (learning speed)

In Figures 6, the learning rate (r) obtained from the learning curve was indicated. In regard to the tactile navigation, significant differences in the hierarchical structure were not observed in terms of learning effects. This can be considered that by combining tactile presentation, a certain learning effect can be expected regardless of the hierarchical structure.

\section{Conditions of favorable hierarchical presentation by tactile interface}

It was found in this experiment that approximately 8-branches/3-layers is the favorable condition of hierarchical presentation in tactile navigation, according to the search time for the first trial and subsequent learning effects. It is important to make the hierarchy structure easy to understand and to memorize in order to produce human-friendly contents. These results are considered as important knowledge in a technique to present a menu screen consisting of items as well as the hierarchical structure in data broadcasting.

\section{Conclusions}

For the barrier-free information receiving system to communicate information provided in digital broadcasting to visually impaired people, we discussed efforts in improving presentation methods from the view point of accessibility. We also evaluated favorable conditions to present hierarchical structure by tactile interface, in regard to items for menu screen or hierarchical structures in data broadcasting. In the experiment, we compared the search time and the number of operations as the evaluation items for the audio navigation and the tactile navigation, mainly from the viewpoint of understanding hierarchical structures. Based on the results, the conditions to present a hierarchy understandable and accessible by visually impaired people were made clearer. However, since the types of hierarchical structures and the number of subjects were limited, further investigation is expected. Since content production and description techniques are so diversified that it is necessary to verify more complicated hierarchical structures and link structures. We also hope to further examine sensory integrative effects as well as effects on short-term memory with touch. We will continue working in order to establish effective presentation methods and access methods for visually impaired people aiming at the information 
presentation environment where everyone is able to receive information on broadcasting and communication.

\section{Acknowledgements}

This study was partially conducted as part of the "Research and development of multimedia browsing technology for visually impaired people", entrusted by The National Institute of Information and Communications Technology (NICT).

\section{References}

1. Sakai, T., Kondoh, S., Matsumura, K., Itoh, T.: Improving Access to Digital Broadcasting for Visually Impaired Users. In: Proceedings of the HCII2005, vol. 7, p. 67 (2005)

2. Ishikawa, J.: Current State and Remaining Issues with Screen Readers for GUI. Transactions of the IPS of Japan 36(12), 1133-1139 (1995)

3. Asakawa, C., Itoh, T.: User Interface for a Non-visual Web Access System. Transactions of the IPS of Japan 40(2), 453-459 (1999)

4. Ebina, T., Igi, S., Miyake, T., Takahashi, H.: GUI Object Search Method by Using a Tactile Display. Transactions of the IEICE of Japan J80-A(11), 2007-2016 (1997)

5. Lee, S.: Access to Computer Systems with Graphical User Interface by Touch. In: Proceedings of the HUMAN FACTORS and ERGONOMICS SOCIETY 39th annual meeting, pp. 742-746 (1995)

6. Aoyagi, S., Hayashi, T., Nakamura, Y., Tondokoro, N.: Accessibility evaluation of website taking into account the use of persons with visual impairments, IEICE of Japan, Technical Report, SP2002-110, WIT2002-50, pp. 9-14 (2002)

7. Umezu, Sagara, Miyagi, Yoda, Psychology Encyclopedia, Heibon co. pp. 85-86 (1981) 